\title{
A Kinetic Model for Pedestrian Evacuation in a Corridor with an Aggressive Sparse Countercurrent ${ }^{\dagger}$
}

\author{
Ana Laura Garcia-Perciante, Marco Antonio Alvarez and Alma Mendez \\ Universidad Autonoma Metropolitana-Cuajimalpa, Ciudad de México, Mexico \\ + Presented at the Entropy 2021: The Scientific Tool of the 21st Century, 5-7 May 2021; Available online: \\ https://sciforum.net/conference/Entropy2021/.
}

Published: 5 May 2021

The modeling of pedestrian flow is a relevant topic which can lead to valuable information for urban planning as well as for improving evacuation strategies. Most works in this field include a heavy numerical component while theoretical predictions are scarce. In the present work we propose a simple yet rich model for bidirectional pedestrian flow in a one dimensional evacuation scenario where a dense crowd of passive walkers exit the building while a sparse group of aggressive individuals attempt to re-enter. The model is based on a kinetic theory treatment with Boltzmann-like equations considering a two moment approach for the transport equations. The corresponding system in linearly analyzed in order to identify stability regions where the flow towards the exit is uninterrupted provided the countercurrent is aggressive enough. The criterion for the onset of a congestion, and thus the relevant parameters in order to avoid it, are obtained in a purely analytical fashion based on statistical physics.

(C) 2021 by the authors. Licensee MDPI, Basel, Switzerland. This article is an open access article distributed under the terms and conditions of the Creative Commons Attribution (CC BY) license (http://creativecommons.org/licenses/by/4.0/). 